Genome Insight

Genomics and Bioinformatics

\title{
Draft genome sequence of Wickerhamomyces anomalus LBCM1105, isolated from cachaça fermentation
}

Aureliano C. Cunha ${ }^{1}$ (D), Renato A. Corrêa dos $\operatorname{Santos}^{2}$ (D), Diego M. Riaño-Pachon ${ }^{3}$ (iD, Fábio M. Squina ${ }^{4}$, Juliana V. C. Oliveira ${ }^{2}$ ID, Gustavo H. Goldman ${ }^{5}$, Aline T. Souza ${ }^{2}$, Lorena S. Gomes ${ }^{1}$, Fernanda Godoy-Santos ${ }^{1}$, Janaina A. Teixeira ${ }^{1}$, Fábio Faria-Oliveira ${ }^{1}$ iD, Izinara C. Rosse ${ }^{1}$, Ieso M. Castro ${ }^{1}$ iD, Cândida Lucas ${ }^{6}$ and Rogelio L. Brandão ${ }^{1}$ iD

${ }^{1}$ Universidade Federal de Ouro Preto, Laboratório de Biologia Molecular e Celular, MG, Brazil.

${ }^{2}$ Centro Nacional de Pesquisa em Energia e Materiais (CNPEM), Laboratório Nacional de Ciência e Tecnologia do Bioetanol (CTBE), Campinas, SP, Brazil.

${ }^{3}$ Universidade de São Paulo, Centro de Energia Nuclear na Agricultura, Laboratório de Biologia

Computacional, Evolutiva e de Sistemas, Piracicaba, SP, Brazil.

${ }^{4}$ Universidade de Sorocaba, Programa de Pós-Graduação em Processos Tecnológicos e Ambientais, Sorocaba, SP, Brazil.

${ }^{5}$ Universidade de São Paulo, Faculdade de Ciências Farmacêuticas, Ribeirão Preto, SP, Brazil.

${ }^{6}$ Universidade do Minho, Centro de Pesquisa Molecular e Ambiental (CBMA), Instituto de Ciência e Inovação para a Bio-Sustentabilidade (IB - S), Braga, Portugal.

\begin{abstract}
Wickerhamomyces anomalus LBCM1105 is a yeast isolated from cachaça distillery fermentation vats, notable for exceptional glycerol consumption ability. We report its draft genome with $20.5 x$ in-depth coverage and around $90 \%$ extension and completeness. It harbors the sequences of proteins involved in glycerol transport and metabolism.
\end{abstract}

Keywords: Non-conventional yeast, glycerol, “de novo" assembly, glycerol.

Received: April 9, 2019; Accepted: April 6, 2020.

Wickerhamomyces anomalus (synonyms Pichia anomala, Hansenula anomala and Candida pelliculosa) are found in several diverse natural habitats, frequently associated with spoilage or processing of food and grain products (Passoth et al., 2006). Different strains of $W$. anomalus were reported (i) to be able to grow on a wide variety of conditions, including different carbon and nitrogen sources (Conceição et al., 2015; Cunha et al., 2019), at both low and high $\mathrm{pH}(2.0$ to 12.4$)$ and from 3 to $37^{\circ} \mathrm{C}$ (Fredlund et al., 2002), (ii) to be highly tolerant to different stress conditions, like osmotic stress (salt), high concentrations of ethanol, and the presence of heavy metals, and (iii) to produce ethanol from glucose, sucrose or xylose. $W$. anomalus strains have also been reported to display constitutive cyanide-resistant alternative oxidase (Cunha et al., 2019). W. anomalus has been used as a cell factory for the production, among others, of enzymes (Díaz-Rincón et al., 2017), biosurfactants (Teixeira Souza et al., 2018) and fermented-beverages (Aplin et al., 2019). Although $W$. anomalus strains show a high industrial

Send correspondence to Diego M. Riaño-Pachon. Universidade de São Paulo, Centro de Energia Nuclear na Agricultura, Laboratório de Biologia Computacional, Evolutiva e de Sistemas, Piracicaba, SP, Brazil. E-mail: email. diego.riano@cena.usp.br versatility, only two strains have its genome sequenced to date (Schneider et al., 2012; Riley et al., 2016).

$W$. anomalus strain LBCM1105 (previously LBCM105) was isolated from sugarcane fermentation vats in a cachaça distillery in Brazil (Conceição et al., 2015), (S22.099694, W41.511090). Extraction of DNA was carried out using the phenol/chloroform method, and purification was performed using the PowerClean DNA Clean-UP kit (MoBio, QIAGEN, Carlsbad, US). The genome size was determined by flow cytometry as previously described (Hare and Johnston, 2011). Cell samples were stained with $2 \mu \mathrm{M}$ Sytox Green (Thermo Fisher Scientific, MA, US) and the assessment was made in triplicate. The genomic library for sequencing was prepared with the Nextera DNA Library kit (Illumina, San Diego, California, US). Genome sequencing (1.0 million paired-end reads of $151 \mathrm{bp}$ ) was performed with an Illumina HiSeq 2500. Quality trimming, and the removal of reads shorter than 90 nucleotides, were carried out using Trimommatic v.0.32 (Bolger et al., 2014). The genome was assembled into contigs ( $20.5 \mathrm{x}$ in depth coverage, $\geq 1 \mathrm{~kb}$ ) using SPAdes v.3.11.1, dipSPAdes mode (Bankevich et al., 2012). The completeness was evaluated by BUSCO v.3.0 (Simão et al., 2015), using the Fungi and Saccharomycetales 
datasets. Genome statistics were computed with QUAST v5.0.2 (Gurevich et al., 2013). A multilocus phylogenetic analysis was performed using RAxML v.8 (Stamatakis, 2014) building a Maximum Likelihood tree based on DNA sequences from the Internal Transcribed Spacers 1 and 2 (ITS1, ITS2), the large and small ribosomal subunits (LSU, $\mathrm{SSU})$, and the Elongation Factor- $1 \alpha(\mathrm{EF}-1 \alpha)$ from species within the genus Barnettozyma, Wickerhamomyces and Candida. The species and the accession numbers of loci LSU, SSU and EF-1 $\alpha$ of the related microorganism were previously described (Kobayashi et al., 2017). The accession numbers for ITS are listed in Figure S1). Saccharomyces cerevisiae S288c was used as the outgroup. The sequences of the loci SSU, LSU and EF-1 $\alpha$ of the LBCM1105 strain were identified via Blast searches using the proper sequences from $W$. anomalus NRRL Y-366 as baits (SSUEF550479.1, LSU- EF550341.1 and EF-1 $\alpha$ - EF552565.1). ITS1 and ITS2 sequences from $W$. anomalus LBCM1105 was extracted using ITSx v.1.0.11 (Bengtsson-Palme et al., 2013). The sequences of ITS1, ITS2, LSU and SSU were aligned using MXSCARNA v.2.1 (Tabei et al., 2008), and of EF-1 $\alpha$ protein using MAFFT v.7 (Katoh et al., 2017). rtREV was selected using IQ-TREE v1.6 (Nguyen et al., 2015) as the best evolutionary model for the EF-1 $\alpha$ phylogenetic analysis. All the alignments were concatenated in a supermatrix using FASconCAT v.1.04 (Kuck and Meusemann, 2010), which was used to conduct a partitioned phylogenetic analysis. A phylogenetic tree based on the alignments and in the evolutionary model (rtREV for EF-1 $\alpha$ and GTR for the others - ITS1, ITS2, LSU and SSU), was inferred using RAxML v.8.4 (Stamatakis, 2014), with 1,000 bootstrap replicates. Genome annotation was done using Augustus v3.3.1 (Stanke et al., 2008) and BRAKER2 v2.1.2 (Hoff et al., 2019), using as extrinsic evidence for training the proteins of $W$. anomalus deposited in GenBank. Proteins related to glycerol transport and metabolism were identified in the LBCM1105 genome using Blastx.

The GC content of the genome was $34.51 \%$. The phylogenetic analysis (Figure S1) confirmed that LBCM1105 is, in fact, a strain within $W$. anomalus, in the same clade with the $W$. anomalus NRRL Y-366-8, with a bootstrap of $100 \%$. Moreover, according to flow cytometry analyses, the genome of strain LBCM1105 is $13.93 \pm 0.11$ $\mathrm{Mb}$. The total genome assembly corresponds to $12.72 \mathrm{Mb}$, i.e., $91.31 \%$ of the expected size, and $89.89 \%$ in relation to the genome of the $W$. anomalus strain NRRL Y-366-8 (GCA 001661255.1) which has a genome size of 14.15 Mb. The completeness of the genome assembly, as evaluated on the gene space by BUSCO, was $88.6 \%$ for the fungi dataset (290 genes) and $85.5 \%$ for the Saccharomycetales dataset (1711 genes). Half of the data is present in 51 scaffolds (L50) larger than $76 \mathrm{~kb}$ (N50), the largest being $229 \mathrm{~kb}$. The total number of contigs was 389 with 6,812 predicted protein-coding genes. This number is similar to the 6,421 ORFs previously reported from the genome of $W$. anomalus NRRL Y-366-8 (Riley et al., 2016), and to the 5,885 ORFs of Saccharomyces cerevisiae (Goffeau et al., 1996). We compared the genome annotation of LBCM1105 (Augustus and BRAKER2) to that of NRRL Y-366-8, S. cerevisiae S288c and $W$. ciferrii using OrthoFinder (Emms and Kelly, 2015). This comparison clearly showed that most predicted genes in LBCM1105 can be assigned to orthologous groups and are shared with the other genomes in the analysis (Figure S2 and Table 1). This Whole Genome Shotgun project has been deposited at DDBJ/ENA/GenBank under the accession SHLV00000000. The version described in this paper is version SHLV01000000.

DNA sequences from $S$. cerevisiae S288c encoding the proteins that perform glycerol transport (the channel Fps $1 p$ and the high affinity transporter Stl1p) and metabolism (the consumption Gut1p/Gut2p, the production Gpd1p/Gpd2p and Gpp1p/Gpp2p, as well as the putative pathway Gcy1p, Ypr1p and Dak1p/Dak2p) (Figure 1, and Table 2) were obtained from SGD (https://www.yeastgenome.org) and used to identify the correspondent putative ORFs in the $W$. anomalus LBCM1105 genome. Homologous sequences to the proteins were found (Table 2), in some cases different $S$. cerevisiae proteins aligned to the same protein in the $W$. anomalus LBCM1105 genome, it is not clear which will be the exact function of the LBCM1105's protein, more studies are need to elucidate this. The $W$. anomalus Stl1p was previously studied in detail, showing very high affinity for glycerol (Cunha et al., 2019). The genome sequence presented here provides evidence for the existence of the genes needed to ensure the two glycerol consumption and production pathways known in $S$. cerevisiae. Further studies are required to verify how intrinsic characteristics of these proteins and their expression and regulation are the cause underlying the LBCM1105's ex-

Table 1 - Comparison of groups of orthologous genes between $W$. anomalus LBCM1105 with two annotation strategies A) Augustus, B) BRAKER2, $W$. anomalus NRRL Y-366-8, W. ciferrii NRRL Y-1031 and S. cerevisiae S288c.

\begin{tabular}{lccccc}
\hline Groups of orthologous genes & LBCM1105-A & LBCM1105-B & S288c & NRRL Y-366-8 & NRRL Y-1031 \\
\hline Number of genes in strains/species & 6812 & 6159 & 6002 & 6421 & 6702 \\
Number of genes in orthogroups & 5965 & 6106 & 4651 & 6227 & 5936 \\
Number of unassigned genes & 847 & 53 & 1351 & 194 & 766 \\
Percentage of genes in orthogroups & 87,6 & 99,1 & 77,5 & 97,0 & 88,6 \\
Number of species-specific orthogroups & 0 & 0 & 7 & 0 & 7 \\
Number of genes in species-specific orthogroups & 0 & 0 & 17 & 0 & 79 \\
\hline
\end{tabular}




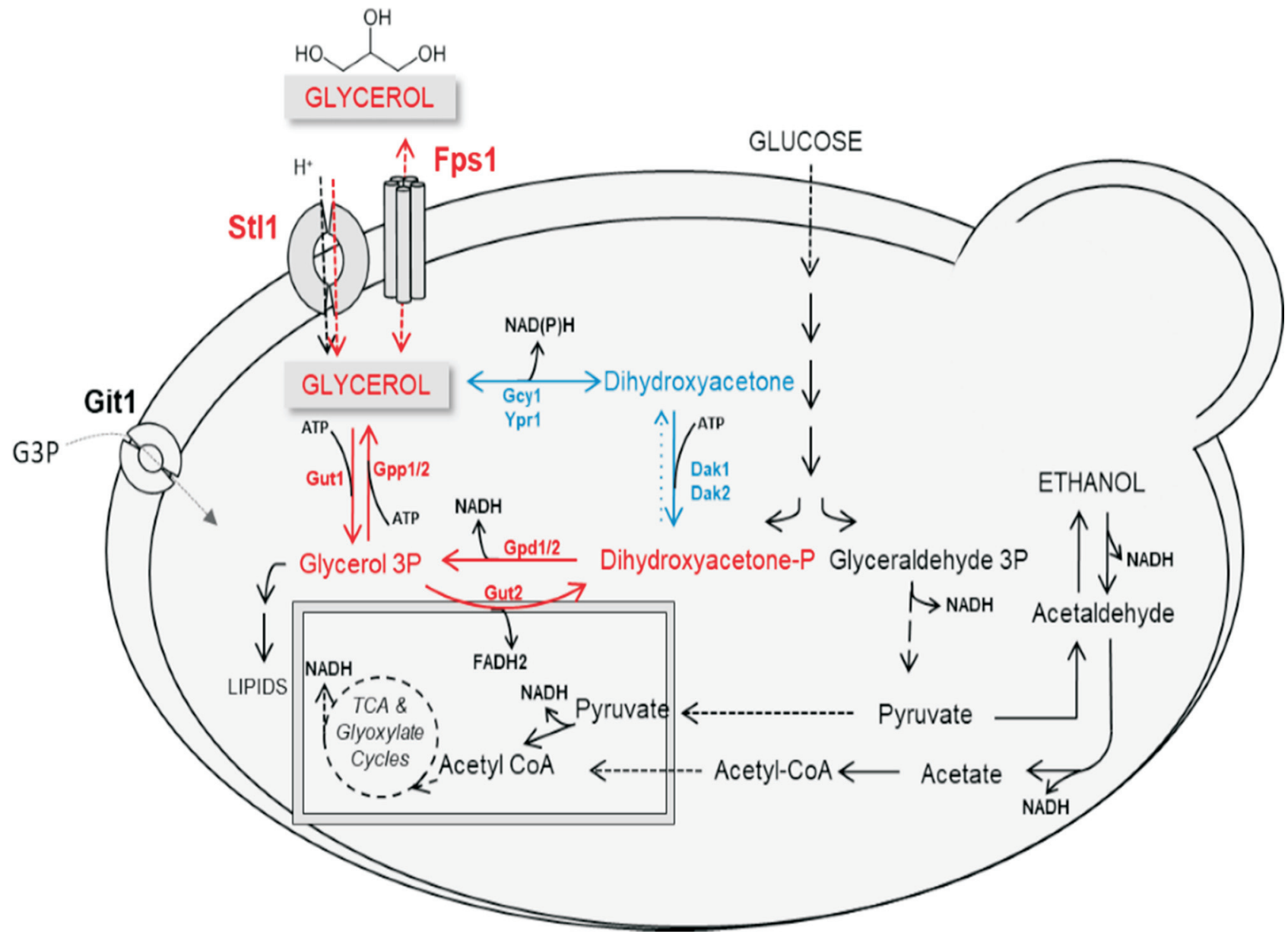

Figure 1 - Global yeast metabolism overview focusing on glycerol transport, consumption and production pathways. Red: main metabolic pathway. Blue: alternative pathway with unclear physiological relevance in S. cerevisiae.

Table 2 - Similarity between the S. cerevisiae genes encoding the proteins responsible for glycerol transport and metabolism as in Figure 1, and the corresponding sequences identified in the genome of $W$. anomalus LBCM1105. Protein Sequences are available at https://doi.org/10.6084/m9.figshare.11441061.v1

\begin{tabular}{|c|c|c|c|c|c|c|c|}
\hline \multicolumn{3}{|c|}{ Protein role } & \multicolumn{2}{|c|}{ S. cerevisiae - SGD database } & \multirow{3}{*}{$\begin{array}{c}\text { Gene } \\
\text { g1373.t1 }\end{array}$} & \multirow{3}{*}{$\begin{array}{c}\begin{array}{c}\text { Percentage } \\
\text { target aligned }\end{array} \\
45.3\end{array}$} & \multirow{3}{*}{$\begin{array}{c}\text { Similarity } \\
56 \%\end{array}$} \\
\hline & & & \multirow{2}{*}{$\begin{array}{l}\text { Gene } \\
\text { FPS1 }\end{array}$} & \multirow{2}{*}{$\frac{\text { ID }}{\mathrm{S} 000003966}$} & & & \\
\hline Regular pathway & Transport & Glycerol channel & & & & & \\
\hline & & $\begin{array}{c}\text { Glycerol active permease/ } \\
\mathrm{H}^{+} \text {symporter }\end{array}$ & STL1 & S000002944 & g4293.t1 & 85.4 & $57 \%$ \\
\hline & Consumption & Glycerol kinase & GUT1 & S000001024 & g1371.t1 & 91.2 & $72 \%$ \\
\hline & & $\begin{array}{c}\text { Glycerol 3P } \\
\text { dehydrogenase/mitochondria }\end{array}$ & GUT2 & S000001417 & g5045.t1 & 98.8 & $72 \%$ \\
\hline & Production & Glycerol 3P dehydrogenase & $G P D 1$ & S000002180 & g1302.t1 & 100 & $78 \%$ \\
\hline & & Glycerol 3P dehydrogenase & GPD2 & S000005420 & g1302.t1 & 81.1 & $82 \%$ \\
\hline & & Glycerol 3P phosphatase & $G P P 1$ & S000002180 & g4575.t1 & 99.2 & $71 \%$ \\
\hline & & Glycerol 3P phosphatase & $G P P 2$ & S000005420 & $\mathrm{g} 4575 . \mathrm{t} 1$ & 99.2 & $71 \%$ \\
\hline \multirow[t]{4}{*}{ Alternative pathway } & Consumption/Production & Glycerol dehydrogenase & $G C Y 1$ & S000005646 & g1045.t1 & 98.7 & $79 \%$ \\
\hline & & Glycerol dehydrogenase & $Y P R 1$ & S000002776 & g1045.t1 & 98.7 & $78 \%$ \\
\hline & Consumption & Dihydroxyacetone kinase & $D A K 1$ & S000004535 & g4297.t1 & 98.5 & $56 \%$ \\
\hline & & Dihydroxyacetone kinase & $D A K 2$ & S000001841 & g4297.t1 & 97.8 & $52 \%$ \\
\hline
\end{tabular}


traordinary ability to grow on glycerol as single a carbon source (Conceição et al., 2015).

\section{Acknowledgments}

The authors gratefully acknowledge Laboratório Nacional de Ciência e Tecnologia do Bioetanol (CTBE) and the Centro Nacional de Pesquisa em Energia e Materiais (CNPEM) for support with the sequencing of LBCM1105. This work was supported by CAPES/Brazil (PNPD 2755/2011; PCF-PVE 021/2012), by CNPq (Brazil), processes 304815/2012 (research grant) and 305135/2015-5, and by AUXPE-PVES 1801/2012 (Process 23038.015294/2016-18) from Brazilian Government and by UFOP. C.L. is supported by the strategic program UID/BIA/04050/2013 [POCI-01-0145-FEDER-007569] funded by national funds through the FCT I.P. and by the ERDF through the COMPETE2020 - Programa Operacional de Competitividade e Internacionalização (POCI). DMRP is a fellow from the CNPq (Conselho Nacional de Desenvolvimento Científico e Tecnológico) - Brazil (310080/2018-5).

\section{Conflict of Interest}

The authors declare that there is no conflict of interest that could be perceived as prejudicial to the impartiality of the reported research.

\section{Authors Contributions}

ACC, LSG, FGS, JAT, FFO, IMC, CL, RLB contributed to project conceptualization; ACC, RACS, DMRP, FMS, JVCO, GHG, ATS, FGS, CL, RLB were responsible for data curation; ACC, RACS, DMRP, FMS, JVCO, GHG, ATS, LSG, FGS, JAT, FFO, ICR, IMC, CL, RLB carried out formal data analysis; DMRP, IMC, CL, RLB were responsible for funding acquisition; ACC, RACS, DMRP, FMS, JVCO, GHG, ATS, LSG, FGS, JAT, FFO, ICR, IMC, CL, RLB performed the experiments, and data collection; ACC, RACS, DMRP, LSG, FGS, JAT, FFO, ICR, CL, RLB designed the methodology; DMRP, IMC, CL, RLB managed and coordinated the project; DMRP, FGS, JAT, FFO, CL, RLB supervised the project; ACC, RACS, DMRP, FGS, FFO, IMC, CL, RLB wrote the original draft; all authors participated in revising and editing the final version of the manuscript.

\section{References}

Aplin JJ, White KP and Edwards CG (2019) Growth and metabolism of non-Saccharomyces yeasts isolated from Washington state vineyards in media and high sugar grape musts. Food Microbiol 77:158-165.

Bankevich A, Nurk S, Antipov D, Gurevich AA, Dvorkin M, Kulikov AS, Lesin VM, Nikolenko SI, Pham S, Prjibelski AD et al. (2012) SPAdes: A new genome assembly algorithm and its applications to single-Cell sequencing. J Comput Biol 19:455-477.
Bengtsson-Palme J, Ryberg M, Hartmann M, Branco S, Wang Z, Godhe A, De Wit P, Sánchez-García M, Ebersberger I, Sousa F et al. (2013) Improved software detection and extraction of ITS1 and ITS2 from ribosomal ITS sequences of fungi and other eukaryotes for analysis of environmental sequencing data. Methods Ecol Evol 4:914-919.

Bolger AM, Lohse M and Usadel B (2014) Trimmomatic: a flexible trimmer for Illumina sequence data. Bioinformatics 30:2114-2120.

Conceição LE, Saraiva MA, Diniz RH, Oliveira J, Barbosa GD, Alvarez F, Correa LF, Mezadri H, Coutrim MX, Afonso RJ et al. (2015) Biotechnological potential of yeast isolates from cachaça: the Brazilian spirit. J Ind Microbiol Biotechnol 42:237-246.

Cunha AC, Gomes LS, Godoy-Santos F, Faria-Oliveira F, Teixeira JA, Sampaio GMS, Trópia MJM, Miranda Castro I, Lucas C and Brandão RL (2019) High-affinity transport, cyanideresistant respiration, and ethanol production under aerobiosis underlying efficient high glycerol consumption by Wickerhamomyces anomalus. J Ind Microbiol Biotechnol 46:709-723.

Díaz-Rincón DJ, Duque I, Osorio E, Rodríguez-López A, EspejoMojica A, Parra-Giraldo CM, Poutou-Piñales RA, Alméciga-Díaz CJ and Quevedo-Hidalgo B (2017) Production of recombinant Trichoderma reesei cellobiohydrolase II in a new expression system based on Wickerhamomyces anomalus. Enzyme Res 2017:6980565.

Emms DM and Kelly S (2015) OrthoFinder: solving fundamental biases in whole genome comparisons dramatically improves orthogroup inference accuracy. Genome Biol 16:157.

Fredlund E, Druvefors U, Boysen ME, Lingsten KJ and Schnurer J (2002) Physiological characteristics of the biocontrol yeast Pichia anomala J121. FEMS Yeast Res 2:395-402.

Goffeau A, Barrell BG, Bussey H, Davis RW, Dujon B, Feldmann H, Galibert F, Hoheisel JD, Jacq C, Johnston M et al. (1996) Life with 6000 genes. Science 274:563-547.

Gurevich A, Saveliev V, Vyahhi N and Tesler G (2013) QUAST: quality assessment tool for genome assemblies. Bioinformatics 29:1072-1075.

Hare EE and Johnston JS (2011) Genome size determination using flow cytometry of propidium iodide-stained nuclei. Methods Mol Biol 772:3-12.

Hoff KJ, Lomsadze A, Borodovsky M and Stanke M (2019) Whole-Genome Annotation with BRAKER. Methods Mol Biol 1962:65-95.

Katoh K, Rozewicki J and Yamada KD (2017) MAFFT online service: multiple sequence alignment, interactive sequence choice and visualization. Brief Bioinform 20:1160-1166.

Kobayashi R, Kanti A and Kawasaki H (2017) Three novel species of d-xylose-assimilating yeasts, Barnettozyma xylosiphila sp. nov., Barnettozyma xylosica sp. nov. and Wickerhamomyces xylosivorus f.a., sp. nov. Int $J$ Syst Evol Microbiol 67:3971-3976.

Kuck P and Meusemann K (2010) FASconCAT: Convenient handling of data matrices. Mol Phylogenet Evol 56:1115-1118.

Nguyen LT, Schmidt HA, von Haeseler A and Minh BQ (2015) IQ-TREE: a fast and effective stochastic algorithm for estimating maximum-likelihood phylogenies. Mol Biol Evol 32:268-274.

Passoth V, Fredlund E, Druvefors UA and Schnurer J (2006) Biotechnology, physiology and genetics of the yeast Pichia anomala. FEMS Yeast Res 6:3-13.

Riley R, Haridas S, Wolfe KH, Lopes MR, Hittinger CT, Goker M, Salamov AA, Wisecaver JH, Long TM, Calvey $\mathrm{CH}$ et al. 
(2016) Comparative genomics of biotechnologically important yeasts. Proc Natl Acad Sci U S A 113:9882-9887.

Schneider J, Rupp O, Trost E, Jaenicke S, Passoth V, Goesmann A, Tauch A and Brinkrolf K (2012) Genome sequence of Wickerhamomyces anomalus DSM 6766 reveals genetic basis of biotechnologically important antimicrobial activities. FEMS Yeast Res 12:382-386.

Simão FA, Waterhouse RM, Ioannidis P, Kriventseva EV and Zdobnov EM (2015) BUSCO: assessing genome assembly and annotation completeness with single-copy orthologs. Bioinformatics 3:3210-3212.

Stamatakis A (2014) RAxML version 8: a tool for phylogenetic analysis and post-analysis of large phylogenies. Bioinformatics 30:1312-1313.

Stanke M, Diekhans M, Baertsch R and Haussler D (2008) Using native and syntenically mapped cDNA alignments to improve de novo gene finding. Bioinformatics 24:637-644.

Tabei Y, Kiryu H, Kin T and Asai K (2008) A fast structural multiple alignment method for long RNA sequences. BMC Bioinformatics 9:33.
Teixeira Souza KS, Gudina EJ, Schwan RF, Rodrigues LR, Dias DR and Teixeira JA (2018) Improvement of biosurfactant production by Wickerhamomyces anomalus CCMA 0358 and its potential application in bioremediation. Toxins 346:152-158.

\section{Supplementary Material}

The following online material is available for this study Figure S1 - Maximum Likelihood (ML) phylogenetic tree based on DNA sequences from large ribosomal subunit (LSU), small ribosomal subunit (SSU) and Elongation Factor- $1 \alpha(\mathrm{EF}-1 \alpha)$.

Figure 5 - Venn Diagram of Groups of Orthologous Genes between $W$. anomalus LBCM1105 (LBCM1105-A: Augustus, LBCM1105-B: BRAKER2), $W$. anomalus NRRL Y366-8, W. ciferrii NRRL Y-1031 and S. cerevisiae S288c.

Associate editor: Ana Tereza Vasconcelos

License information: This is an open-access article distributed under the terms of the Creative Commons Attribution License (type CC-BY), which permits unrestricted use, distribution and reproduction in any medium, provided the original article is properly cited. 